EPJ Web of Conferences 79, 03011 (2014)

DOI: $10.1051 /$ epjconf/20147903011

(C) Owned by the authors, published by EDP Sciences, 2014

\title{
Concentrated photovoltaics, a case study
}

\author{
Piergiorgio Antonini ${ }^{1,2, a}$, Sandro Centro ${ }^{1,2,3}$, Stelvio Golfetto ${ }^{3}$ and Alessandro Saccà ${ }^{2,4}$ \\ ${ }^{1}$ Centro Fermi, P.zza del Viminale 1, 00184 Roma, Italy \\ ${ }^{2}$ Physics and Astronomy Department, University of Padova, via Marzolo 8, 35131 Padova, Italy \\ ${ }^{3}$ AtemEnergia srl, Gall. Borromeo 3, 35137 Padova, Italy \\ ${ }^{4}$ Department of Industrial Engineering, University of Padova, via Venezia 1, 35131 Padova, Italy
}

\begin{abstract}
Concentrated Photovoltaics (CPV), once a niche technology, has now reached the maturity and reliability for large scale power generation. Especially in regions where temperatures are very high, the use of high efficiency triple junction solar cells with concentrating optics allows stable energy yield. Thus CPV can be seen as complementary and not in concurrence with silicon photovoltaics. The state of the art, the advantages and limitations of this technology will be shown. Among the main advantages of CPV is the possibility of a much higher energy supply, when compared to silicon photovoltaics, both comparing CPV and silicon with same area or the same installed power. The use of recycled and recyclable materials allows a more environmentally friendly production. The possibility to couple CPV with desalination facilities, energy storage will be analysed. As an example a case study of a CPV installation in Northern Italy is discussed. Here the use of mature technologies, derived from automotive and lighting sectors resulted in a simple and efficient module.
\end{abstract}

\section{Concentrated photovoltaics, definitions and motivations}

Concentrated photovoltaics is based on the simple idea of using low cost materials such as plastic to concentrate the sun light on very high efficiency solar cells.

The use of optical elements to concentrate the sun light is convenient only using very high efficiency solar cells. Thus research on concentrated photovoltaics, a very old idea [1], had a real boost only when multiple junctions solar cells could be sold at acceptable costs. Multiple junctions solar were first developed for the powering of satellites, first double junctions, and then triple junctions (now a standard technology). Triple junction (3J) solar cells have now (2013) overcome the efficiency of $44 \%$, and 4 to 5 -junctions solar cells are expected to reach $50 \%$ of efficiency.

Concentrated photovoltaics module can be divided in two main layouts: having the concentrating optics in lenses (usually Fresnel lenses) or in mirrors, shown in Fig. 1. Fresnel lenses can be of moulded PMMA or silicon-on-glass (a thin film of silicon glued to a flat glass); mirror can be made of glass or plastic covered by a thin layer of aluminium or silver then protected against oxidation. There are many different considerations that lead the designer toward one layout or the other. The main differences

\footnotetext{
${ }^{\text {a }}$ Corresponding author: antonini@pd.infn.it
}

This is an Open Access article distributed under the terms of the Creative Commons Attribution License 4.0, which permits unrestricted use, distribution, and reproduction in any medium, provided the original work is properly cited. 


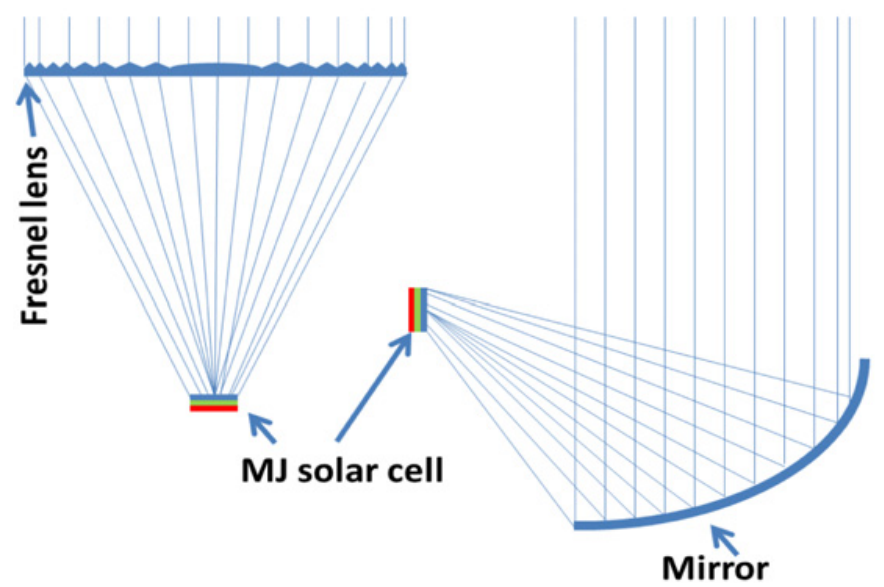

Figure 1. A concentrated photovoltaics optical layout can be either made with lenses (usually Fresnel lenses) or mirrors. Fresnel lenses can be of moulded PMMA or silicon-on-glass (a thin film of silicon glued to a flat glass); mirror can be on glass of plastic covered by a thin layer of aluminium or silver then protected against oxidation.

are that lenses suffer of chromatic aberration that makes it more difficult to design a homogeneous illumination profile. Mirrors do not have chromatic aberration but great care must be dedicated to the choice of the reflective layer to avoid oxidation or other optical losses.

Either way, concentrating optics must provide a very homogenous illumination profile on the solar cell. Failing this can lead to efficiency and reliability problems. At the same time the optics must be robust enough to ensure that even when the module is misaligned (i.e. not pointing to the Sun) enough light is still reaching the solar cell. These two goals can be reached simultaneously using nonimaging optics [2].

Concentrated photovoltaics and the more traditional "flat" photovoltaics (FPV) (the usually silicon based modules that are often on rooftops or fields) are two technologies that are not in competition, they are more complementary. This can be understood analysing the main differences between them.

Flat PV has the remarkable plus of being a very simple technology, reliable and cheap. Nevertheless, FPV modules, based on silicon solar cells, have low efficiency, thus requiring large areas for their installation. Moreover, the efficiency of silicon solar cells is highly dependent on temperature $(-0.3 \% / \mathrm{K})$. For this reason silicon PV modules perform badly in areas where there is a high irradiation, where usually air temperatures are very high.

Concentrated photovoltaics modules use $3 \mathrm{~J}$ solar cells, whose temperature coefficient is almost one order of magnitude lower $(-0.04 \% / \mathrm{K})$ [3]. They are thus performing very well also in hot climates, even using passive (air) cooling $[4,5]$. The use of higher efficiency solar cells allows a CPV module to produce more power and energy using less area. CPV modules can also be made of recyclable materials, improving the overall footprint.

The use of dual axis sun trackers give also the possibility to use the land under the modules, reducing to almost nothing the areas occupied by the CPV modules.

\section{Twinfocus, a case study on concentrated photovoltaics}

A research funded by Centro Fermi and University of Padova led to the development of a concentrated photovoltaics module, called TwinFocus due to its symmetrical configuration of two mirrors concentrating the solar light onto two opposite $3 \mathrm{~J}$ solar cells $[6,7]$. The system is described in detail in the contribution of A. Saccà in this same volume [8]. 


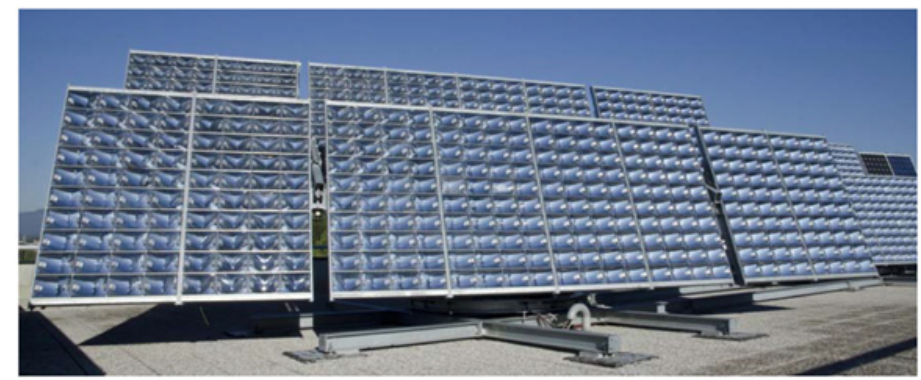

Figure 2. The sun tracker is made of 640 TwinFocus concentrators and has a closed-loop tracking control. Four such trackers have been mounted at the premises of three private companies collaborating to this project. A fourth tracker has been installed by Centro Fermi and the University of Padova. All of them are installed in Northern-East of Italy.

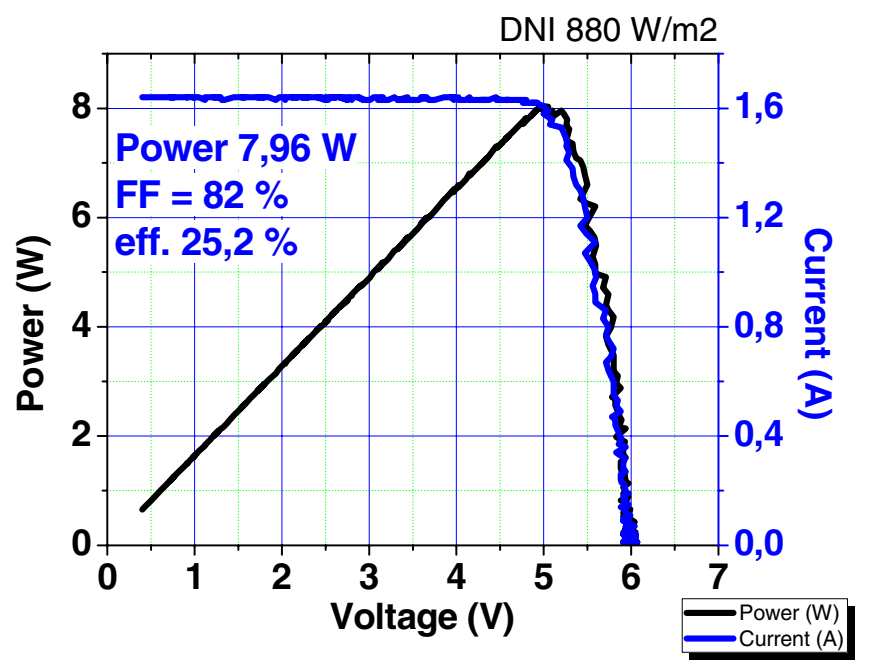

Figure 3. Current-Voltage (IV) curve of a TwinFocus module, measured near CSOC conditions (DNI $880 \mathrm{~W} / \mathrm{m}^{2}$ instead of $900 \mathrm{~W} / \mathrm{m}^{2}$ ). The measured efficiency is of $25.2 \%$.

The concentrators, with a concentrating ratio of 580X, are connected together to form a $4,64 \mathrm{kWp}$ sun tracker. It is shown in Fig. 2. Four sun trackers have been installed in Northern-East of Italy, threeby private companies collaborating to the project, and a fourth one by Centro Fermi and the University of Padova.

The efficiency of a concentrated photovoltaics module (and system) depends on weather conditions. There is accordance to the fact that the efficiency (and consequently power) of the system must be rated at CSOC condition. CSOC condition means: Direct Normal Irradiance (DNI) of $900 \mathrm{~W} / \mathrm{m}^{2}$, Air Mass 1.5 , air temperature $25^{\circ} \mathrm{C}$ and wind speed $2 \mathrm{~m} / \mathrm{s}$. In these conditions the best concentrator showed an efficiency of more than $25 \%$. The corresponding current-voltage (I-V) curve is showed in Fig. 3.

The system efficiencies of the four systems varied in the range 22-24\%. The system efficiency is lower than single concentrator efficiency due to the fact that the concentrators show differences in efficiency because of:

- different thickness in reflective layers causing different reflectance;

- differences in components mounting causing misalignments; 


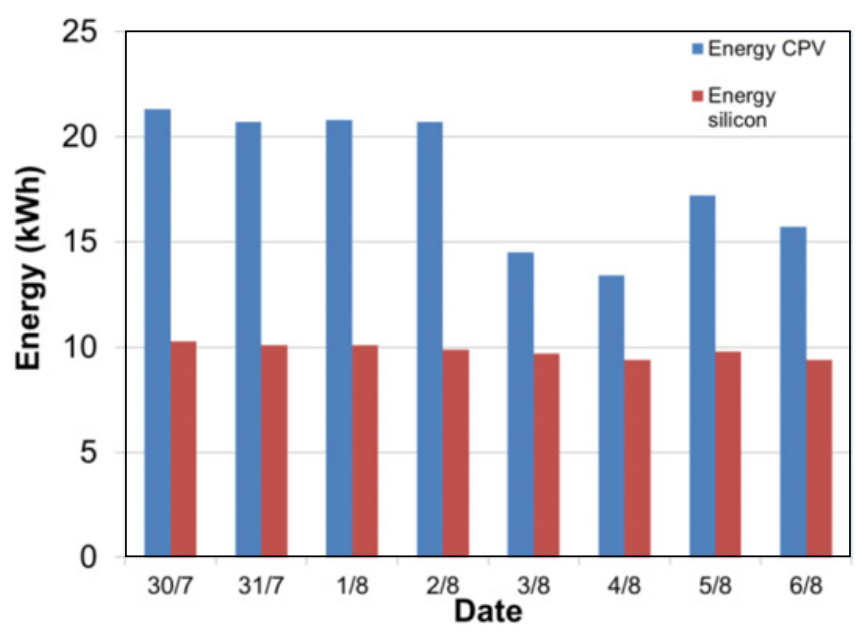

Figure 4. Energy production of CPV (blue bars) and silicon PV (red bars) during a Summer week in Northern Italy. The energy production was almost double for CPV, and more than double during the first four days.

- differences in components mounting causing mismatch in thermal heat sinks.

All these aspects have been identified. They are mainly caused by the fact that the concentrators have been constructed manually, not industrially.

Another aspect that justifies the lower system efficiency compared to single concentrator efficiency is called system mismatch: different IV curves between the concentrators cause the fact that the maximum power point is not the same for all concentrators, and the working point, decided by the DC/AC inverter, is not optimized for all 3J solar cells. This is common to all photovoltaic system and cannot be avoided. It can only be minimized obtaining that all concentrator be very similar to each other.

Figure 4 shows an example of a week of measurements between the end of July and the begin of August, in San Vendemiano, about $60 \mathrm{~km}$ north of Venice, Italy. The peak air temperature on those days was in average around $32^{\circ} \mathrm{C}$, wind almost absent. The measurement is interesting because it shows how the system performs, compared to polycrystalline silicon flat PV with a measurement on clear and hot days. In fact, as mentioned above, CPV is the best solar technology on very hot climates, different from Northern Italy that is rather temperate. It can be seen that already in this not extreme weather condition comparing the same areas for CPV and flat PV the energy production is almost double for CPV: $144 \mathrm{kWh}$ for CPV and $78 \mathrm{kWh}$ for flat PV. It can also be seen that during the first four days (when the weather was better, with total absence of clouds), the energy production of CPV was effectively twice the flat PV energy production.

\section{Concentrated photovoltaics and storage systems}

In a concentrated photovoltaic system the use of a dual axis sun tracker is necessary. This is a drawback, introducing a cost and a loss of reliability. Is also true, on the other hand, that the presence of a sun tracker gives to CPV systems a power curve that fits better with smart use of the energy and storage. This is due to the fact that following the sun path, the CPV modules are always normal to the sun rays direction, and thus almost immediately after the sunrise providing the maximum power, lasting almost until dusk. In opposite, the power given by fixed photovoltaic modules is modulated by the sinus law, so that only around noon the power corresponds to the rated power of the system. This is explained in Fig. 5. The curves are measured in the system in San Vendemiano, Northern Italy. The red line is the power of the polycrystalline-silicon $240 \mathrm{Wp}$ flat PV modules, the green line that of similar modules 


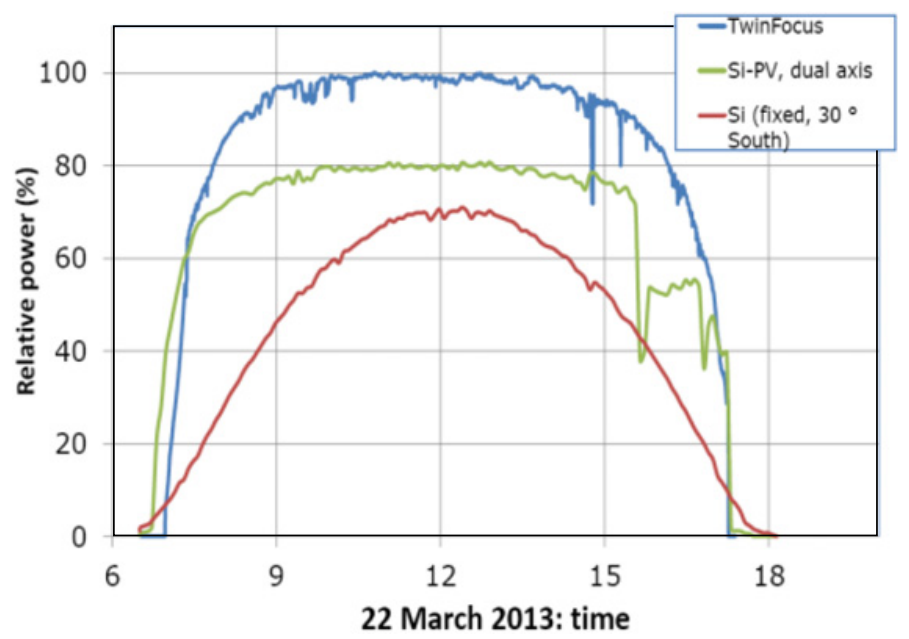

Figure 5. Energy production of CPV (blue line) and silicon PV (red line for fixed, green for dual axis tracking) during a Spring day in Northern Italy. The power loss in the last part of the green line is due to shading. The availability of more power in the first and last hours of the day allow for a lower power and energy capacity of storage (if needed).

mounted in a second sun tracker (of the same model of the CPV system), the blue line the power of the CPV system. The three systems are of the same area. The decrease in power of the green line in the late afternoon is due to shading and is not relevant for the discussion carried here.

It can be seen that the power of the CPV system reached it maximum before 9:00 AM, already accumulating a large amount of energy (relative power are in vertical axis, rating $100 \%$ the maximum of COV that day). With such a curve, if storage is needed, a smaller amount of energy must be stored for example for night use, because there is already large energy production early in the morning, and late in the afternoon. The availability of large amount of energy for a wider time interval allows for use of batteries of smaller power and energy capacity. This is important, because both power and energy capacity affect the cost of a storage system

The reduction of the feed-in tariffs that paid the energy produced with photovoltaics systems made more urgent the use of storage systems. In fact without a storage system, the only possibility for the owner of a photovoltaic system to sue the value of the energy produced it to sell it to the energy provider (the "utility"). The problem here is that the utility pays much less (usually more or less on half) the energy it buys compared to the price of the energy it sells. So, if the same amount of energy is later needed, at the end the value of the photovoltaic energy produced is helved. To avoid this one solution seems to be storage, but the problem is that storage systems are costly. And the cost is proportional to the energy and/or power capacity. Self-consumption is then a better way. Self-consumption is easier if the power provided by the PV system is more constant (like in CPV).

Another way to exploit a CPV system in a good trade-off between storage and self-consumption is electric mobility. The principle here is that in one hand using CPV system the electric car can be charged during a larger span of time, thanks to the more regular power curve, and on the other hand the presence of a stack of batteries in the electric cars represent the storage system that so it is not necessary to buy.

As an example, a new generation TwinFocus system of $14 \mathrm{kWp}$ can provide more or less $15000 \mathrm{kWh} /$ year. This value strongly depends on location and it is taken as an example in Northern Italy. In Southern Europe this value can also be doubled. Considering a standard electric car, that needs $15 \mathrm{kWh} / 100 \mathrm{~km}$, this means that this CPV system can feed a number of e-cars for a total of 
$100000 \mathrm{~km} /$ year (for instance 5 e-car running $20000 \mathrm{~km} /$ year each). This corresponds to the saving of 5000 litres of petrol/year.

Another smart way to store energy without buying batteries is the production of clean water. A reverse osmosis (RO) system needs (depending on size) more or less 2.5 to $3 \mathrm{kWh} / \mathrm{m}^{3}$ of clean water. This means that a $14 \mathrm{kWp}$ CPV system giving in a Summer day (more or less 10 hours of Sun) $140 \mathrm{kWh}$, can provide enough energy for a RO system to produce $50 \mathrm{~m}^{3}$ of clean water, enough for the daily need of 250 people. This is a way to store the value of the energy (as valuable clean water) rather than the energy itself.

\section{Conclusions}

Concentrated Photovoltaics is a reliable and high efficient technology. The performances of TwinFocus, a concentrated photovoltaics module and system developed have been presented. The research and development was funded by Centro Fermi, University of Padova, Regione Veneto (Italy) and a group of four private companies, one of these being AtemEnergia, a spin-off company of the University of Padova.

\section{References}

[1] R. M. Swanson, Progress in Photovoltaics: Research and Applications, 8, 93-111 (2000)

[2] R. Winston, J. C. Miñano, P. Benítez: Nonimaging Optics, Elsevier (2005)

[3] G. S. Kinsey, P. Hebert, K. E. Barbour, D. D. Krut, H. L. Cotal R. A. Sherif, Progress in Photovoltaics: Research and Applications, 16, 503-508 (2008)

[4] A. Luque, G. Sala, J.C. Arborio: Solar Energy Materials and Solar Cells 51, 269-290 (1998)

[5] A. Royne, Ch. J. Dey, D. R.Mills: Solar Energy Materials and Solar Cells 86, 451-483 (2005)

[6] P. Antonini, S. Centro, S. Golfetto, A. Saccà, SPIE Newsroom (2013) DOI: $10.1117 / 2.1201303 .004779$

[7] P. Antonini, S. Centro, S. Golfetto, A. Saccà, Il Nuovo Saggiatore 29 1-2, pp 39-50 (2013)

[8] P. Antonini, S. Centro, S. Golfetto, A. Saccà, EPJ Web of Conferences, next contribution 